\title{
Combined Neuropathological Pathways Account for Age-Related Risk of Dementia
}

\author{
Melinda C. Power, ScD, ${ }^{1}$ Elizabeth Mormino, $\mathrm{PhD}{ }^{2}$ Anja Soldan, $\mathrm{PhD},{ }^{3}$ \\ Bryan D. James, PhD, ${ }^{4,5}$ Lei Yu, PhD, ${ }^{6}$ Nicole M. Armstrong, PhD, ${ }^{7}$ \\ Katherine J. Bangen, PhD, ${ }^{8,9}$ Lisa Delano-Wood, PhD, 8,9 Melissa Lamar, PhD, 4,10 \\ Yen Ying Lim, PhD, ${ }^{11}$ Kelly Nudelman, PhD, ${ }^{12}$ Laura Zahodne, PhD, ${ }^{13}$ \\ Alden L. Gross, MHS, PhD, ${ }^{7,14,15}$ Dan Mungas, PhD, ${ }^{16}$ Keith F. Widaman, PhD, ${ }^{17}$ and \\ Julie Schneider, MD $4,6,18$
}

Objective: Our objectives were to characterize the inter-relation of known dementia-related neuropathologies in one comprehensive model and quantify the extent to which accumulation of neuropathologies accounts for the association between age and dementia.

Methods: We used data from 1,362 autopsied participants of three community-based clinicopathological cohorts: the Religious Orders Study, the Rush Memory and Aging Project, and the Minority Aging Research Study. We estimated a series of structural equation models summarizing a priori hypothesized neuropathological pathways between age and dementia risk individually and collectively.

Results: At time of death (mean age, 89 years), $44 \%$ of our sample had a clinical dementia diagnosis. When considered individually, our vascular, amyloid/tau, neocortical Lewy body, and TAR DNA-binding protein 43 (TDP-43)/hippocampal sclerosis pathology pathways each accounted for a substantial proportion of the association between age and dementia. When considered collectively, the four pathways fully accounted for all variance in dementia risk previously attributable to age. Pathways involving amyloid/tau, neocortical Lewy bodies, and TDP-43/hippocampal sclerosis were interdependent, attributable to the importance of amyloid beta plaques in all three. The importance of the pathways varied, with the vascular pathway accounting for $32 \%$ of the association between age and dementia, wheraes the remaining three inter-related degenerative pathways together accounted for 68\% (amyloid/tau, 24\%; the Lewy body, $1 \%$; and TDP-43/hippocampal sclerosis, 43\%).

Interpretation: Age-related increases in dementia risk can be attributed to accumulation of multiple pathologies, each of which contributes to dementia risk. Multipronged approaches may be necessary if we are to develop effective therapies.

ANN NEUROL 2018;84:10-22

View this article online at wileyonlinelibrary.com. DOI: 10.1002/ana.25246

Received Nov 2, 2017, and in revised form Apr 18, 2018. Accepted for publication Apr 20, 2018.

Address correspondence to Dr Melinda C. Power, George Washington University Milken Institute School of Public Health, 950 New Hampshire Avenue Northwest, Washington, DC 20052. E-mail: Power@gwu.edu

From the ${ }^{1}$ Department of Epidemiology and Biostatistics, George Washington University Milken Institute School of Public Health, Washington, DC; ${ }^{2}$ Department of Neurology, Massachusetts General Hospital, Harvard Medical School, Boston, MA; ${ }^{3}$ Department of Neurology, Johns Hopkins University School of Medicine, Baltimore, MD; ${ }^{4}$ Rush Alzheimer's Disease Center, Rush University, Chicago, IL; ${ }^{5}$ Department of Internal Medicine, Rush University, Chicago, IL; ${ }^{6}$ Department of Neurological Sciences, Rush University, Chicago, IL; ${ }^{7}$ Department of Epidemiology, Johns Hopkins Bloomberg School of Public Health, Baltimore, MD; ${ }^{8}$ VA San Diego Healthcare System, San Diego, CA; ${ }^{9}$ Department of Psychiatry, University of California San Diego, San Diego, CA; ${ }^{10}$ Department of Behavioral Sciences, Rush University Medical Center, Chicago, IL; ${ }^{11}$ Florey Institute of Neuroscience and Mental Health, Parkville, VIC, Australia; ${ }^{12}$ Department of Radiology and Imaging Sciences, Indiana University-Purdue University at Indianapolis, Indianapolis, IN;

${ }^{13}$ Department of Psychology, University of Michigan, Ann Arbor, Ml; ${ }^{14} \mathrm{~J}$ ohns Hopkins Center on Aging and Health, Baltimore, MD; ${ }^{15}$ Department of Mental Health, Johns Hopkins Bloomberg School of Public Health, Baltimore, MD; ${ }^{16}$ Department of Neurology, University of California-Davis, Davis, CA;

${ }^{17}$ Graduate School of Education, University of California Riverside, Riverside, CA; and ${ }^{18}$ Department of Pathology, Rush University Medical Center, Chicago, IL

Additional supporting information may be found online in the Supporting Information section at the end of the article. 
$\mathrm{M}$ ultiple age-related neuropathologies are increasingly recognized as contributors to dementia risk. ${ }^{1-6}$ Although dementia is most commonly associated with Alzheimer's disease (AD) neuropathology, namely amyloid-beta plaques and neurofibrillary tangles, pure $\mathrm{AD}$ is relatively rare; the majority of dementia cases clinically diagnosed with probable $\mathrm{AD}$ show evidence of multiple neuropathologies. ${ }^{4,7-11}$ Even among pathologically confirmed $\mathrm{AD}$ cases, significant comorbidities are common and are frequently associated with worse clinical outcomes. For instance, for a given level of $\mathrm{AD}$ neuropathology, the presence of infarcts ${ }^{12}$ or cortical microinfarcts ${ }^{13}$ is associated with greater cognitive impairment, suggesting that underlying vascular disease increases dementia risk independent of $\mathrm{AD}$ neuropathology. Cerebral amyloid angiopathy (CAA), which commonly coexists with $\mathrm{AD}$ pathology and which is associated with other features of cerebrovascular disease, ${ }^{14}$ is also independently associated with cognitive impairment and dementia. ${ }^{15,16}$ Although Lewy bodies can contribute to dementia risk in the absence of $\mathrm{AD}$ neuropathology, Lewy bodies are common in persons with pathologically confirmed $\mathrm{AD},{ }^{17,18}$ and individuals with both $\mathrm{AD}$ pathology and Lewy bodies show greater risk of cognitive decline or dementia. ${ }^{19-21}$ Likewise, TAR DNA-binding protein 43 (TDP-43) aggregations are common in aging, in pathologically confirmed $\mathrm{AD}$, and in combination with hippocampal sclerosis, ${ }^{22,23}$ and TDP-43 aggregations increase the likelihood of dementia, including a clinical diagnosis of $\mathrm{AD} .^{7,24}$

The extent to which these neuropathological pathways inter-relate and whether known neuropathologies can fully account for age-related increases in dementia risk is currently unknown. A fuller understanding will be critical to the development and targeting of effective therapies. Using structural equation modeling (SEM), we tested the significance and inter-relations of all major dementiarelated neuropathologies by which age was hypothesized to elevate dementia risk in an autopsy sample of persons recruited from the community.

\section{Patients and Methods}

\section{Study Sample}

We used clinical and neuropathological data from autopsied participants of three Rush Alzheimer's Disease Center clinical-pathological cohorts of aging: the Religious Orders Study (ROS), ${ }^{25}$ the Rush Memory and Aging Project (MAP), ${ }^{26}$ and the Minority Aging Research Study (MARS). ${ }^{27}$ Briefly, ROS began in 1994 and recruited older Catholic nuns, priests, and brothers from religious orders across the United States. Participants were enrolled from over 40 groups and included communally living employed and retired persons. MAP began in 1997 and recruited older adults living in retirement communities and subsidized housing facilities across the Chicago metropolitan area. MARS began in 2004 and recruited older adults who self-identified as African American or black from churches, subsidized senior housing facilities, retirement communities, clubs, and organizations in the Chicago metropolitan area. Recruitment efforts involved relationship building with local communities and consultation with community-based advisory groups. These three cohorts are designed to allow combined analyses. For all three, eligibility criteria required absence of recognized dementia at recruitment and agreement to annual clinical evaluations and cognitive testing. Participation in ROS and MAP required brain donation at death. MARS participants were asked, but not required, to donate starting in 2010. At the time of writing, the three studies had enrolled 4,094 subjects, and follow-up rates exceeded $90 \%$ of survivors. All three studies were approved by the institutional review board of Rush University Medical Center. All participants in our sample signed an informed consent and an Anatomical Gift Act form.

At the time of analyses, 1,744 participants had died and autopsies had been completed on 1,418 (81.3\%) participants. Our sample consists of the 1,362 persons with valid neuropathology data and a final consensus cognitive diagnosis of cognitive status before death.

\section{Measures}

Age at death was based on self-reported date of birth and known date of death. Cognitive diagnosis proximate to death was determined by a neurologist with expertise in dementia who reviewed the available clinical data (ie, interview data, medical history and examination data, neuropsychological testing, and annual diagnostic classification). For difficult cases, diagnosis was ascertained by consensus at a case conference including at least one neurologist and one neuropsychologist. All cognitive diagnoses were made blinded to postmortem data. AD dementia was classified according to NINCDS-ADRDA criteria ${ }^{28}$; lesscommon dementia subtypes were classified according to accepted criteria or contemporary standards. ${ }^{29-31}$ For this analysis, Individuals with a dementia diagnosis according to any dementia criteria were classified as having dementia.

Brain autopsies were standardized and completed by study personnel blinded to clinical information. ${ }^{4,32,33}$ Briefly, each brain hemisphere was cut in coronal 1-inch slabs. One hemisphere was fixed in $4 \%$ paraformaldehyde and then cut, whereas the other was immediately photographed and frozen. Regions of interest were dissected from the fixed slabs and embedded in paraffin; sections of 
the paraffin blocks were cut and stained to characterize pathologies. All measures were reviewed by a boardcertified neuropathologist.

Neuritic plaques and neurofibrillary tangles (tangles) were counted during microscopic examination of Bielschowsky silver-stained 6-micron sections on slides from five regions (hippocampus, midfrontal cortex, midtemporal cortex, inferior parietal cortex, and entorhinal cortex). Total number was counted within a $1-\mathrm{mm}^{2}$ area of apparent greatest density of each index. We scaled regional counts of neuritic plaques and tangles by dividing the count of each region by the corresponding standard deviation. Scaled regional counts of neuritic plaques were averaged to provide a single neuritic plaques summary measure. Latent variable summaries of mesiotemporal and neocortical tangles were derived from scaled regional counts of tangles for use in analyses. Fixed slabs or photographs were evaluated for evidence of gross infarcts; we quantified gross infarcts as counts of visualized, dissected, and histologically confirmed chronic macroscopic infarcts. Paraffin-embedded sections from a minimum of nine brain regions stained with hematoxylin/eosin (midfrontal cortex, middle temporal cortex, inferior parietal cortex, entorhinal cortex, hippocampus cortex, basal ganglia, thalamus, and midbrain) were used to characterize burden of microinfarcts defined as infarcts identified by microscopy, but not gross visual examination. We considered the count of ascertained microinfarcts across all sections. Macroscopic and microscopic infarct counts were parameterized as $0, \quad 1, \quad$ or $\geq 2$ infarcts. Neuropathologists characterized cerebral atherosclerosis, arteriolosclerosis, and CAA using an ordinal scale ranging from 0 (none) to 3 (severe). Ratings of atherosclerosis were based on visual examination of the circle of Willis, arteriolosclerosis was quantified based on evaluation of the small vessels of the anterior basal ganglia, and CAA was assessed based on evaluation of paraffin-embedded sections of four neocortical regions (midfrontal, middle temporal, inferior parietal, and occipital) immunostained for beta-amyloid (antibody; Covance Labs [Princeton, NJ], Dako [Carpinteria, CA], or Elan Pharmaceuticals [Dublin, Ireland]). ${ }^{32}$ Lewy body disease was ascertained as present, brainstem-predominant, limbic-type, or neocortical-type based on evaluation of alpha-synuclein immunostained sections (antibody; Zymed Laboratories [South San Francisco, CA] or Wako Chemicals USA, Inc. [Richmond, VA]) from multiple brain regions and a modified version of the McKeith criteria. ${ }^{34}$ We considered only the presence or absence of neocortical Lewy body disease, because only neocortical Lewy bodies appear related to dementia. ${ }^{35}$ Severity of TDP-43 was quantified across six brain regions (amygdala, hippocampus CA1, hippocampus dentate, entorhinal cortex, middle temporal cortex, and midfrontal cortex) based on the number of cytoplasmic inclusions observed in the $0.25-\mathrm{mm}^{2}$ area of greatest density after immunostaining for monoclonal antibodies to phosphorylated TDP-43 (antibody; Ascension Laboratories, Gaithersburg, $\mathrm{MD})$. Each region was reviewed for neuronal and glial inclusions and rated on an ordinal 6-point scale. Total TDP-43 severity was quantified as the mean rating across the six regions. Hippocampal sclerosis was detected using hematoxylin and eosin staining and the presence of severe neuronal loss in CA1 hippocampal subfield and/or subiculum. See http://www.radc.rush.edu/docs/var/variables/htm for additional details.

\section{Statistical Analysis}

We used SEM to estimate models including both latent and manifest variables that summarized our a priori hypothesized neuropathological pathways between age and dementia risk, described below. These analyses tested whether our hypothesized models were consistent with the data and estimated the strength and significance of each hypothesized relation in our model. We estimated all parameters using weighted least squares with standard errors and mean- and variance-adjusted test statistics with a full weight matrix (WLSMV), which yields parameter estimates and standard errors that are robust to violations of multivariate normality. This method yielded measures of model fit and allowed use of data from all participants, even if data on certain variables were missing for some participants. Because several variables in our models were categorical, we report standardized parameter estimates from WLSMV estimation to enable more direct comparisons of magnitude of effects for different pathways to dementia. We used the $\chi^{2}$ statistic and several practical fit indices when evaluating model fit to data, including the root mean square error of approximation (RSMEA), comparative fit index (CFI), Tucker-Lewis index (TLI), and standardized root mean square correlation (SRMR). An RSMEA $\leq 0.05$, CFI $>0.95$, TLI $>0.95$, and SRMR $<$ 0.08 indicate close model fit to data. ${ }^{36}$

To begin, we used confirmatory factor analysis (CFA) to model latent variables summarizing a subset of pathologies. A priori, we hypothesized a latent variable for (1) vessel disease, informed by measures of arteriolosclerosis and atherosclerosis, (2) infarcts, informed by measure of gross and microcerebral infarcts, (3) neocortical tangles, informed by tangles in the inferior parietal, midfrontal, and midtemporal cortices, and (4) mesiotemporal tangles, informed by tangles in the entorhinal and hippocampus cortices. Assessment of model fit supported a modification allowing midtemporal tangles counts to inform both the neocortical and mesiotemporal tangles latent constructs. 


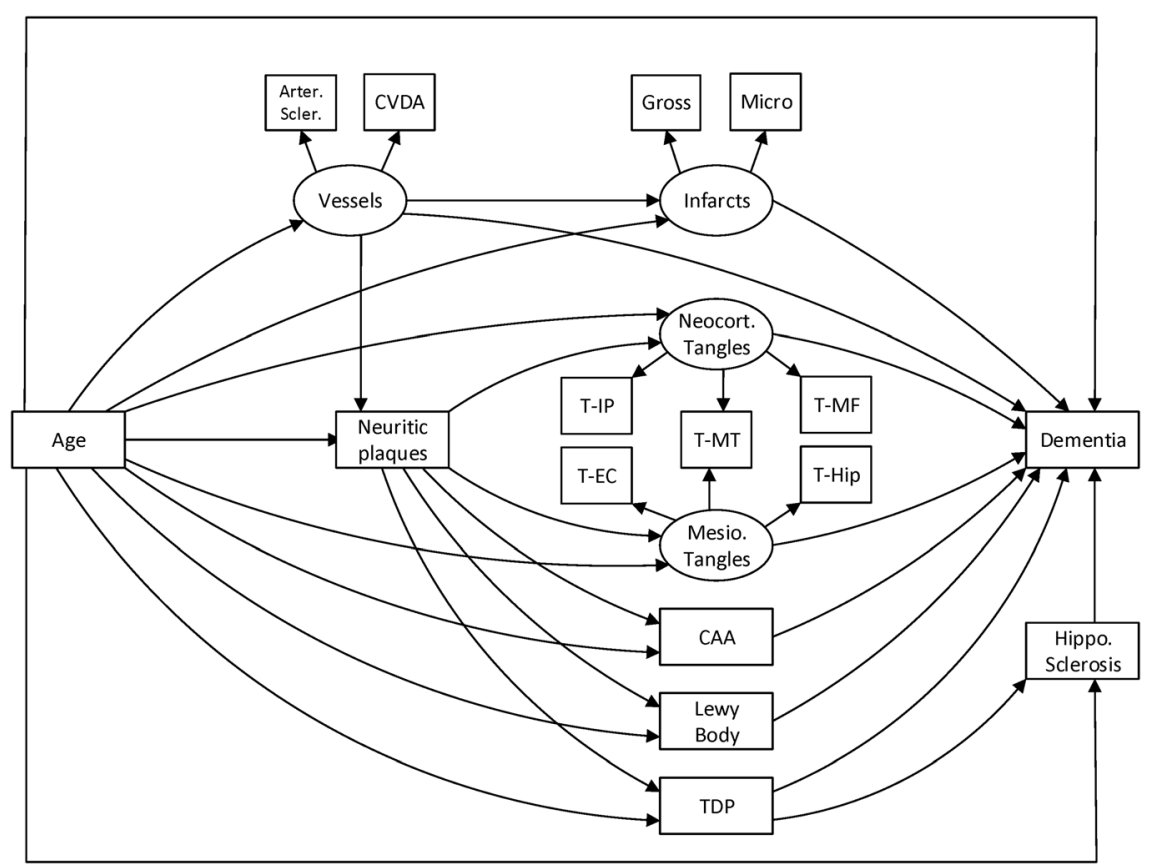

FIGURE 1: Hypothesized pathways by which neuropathology may mediate the association of age and dementia. Figure represents our a priori hypothesized model, with the addition of a modification allowing midtemporal tangles to contribute to the neocortical tangles latent variable. Squares or rectangles represent manifest variables and ellipses represent latent variables. Each single-headed arrow denotes a hypothesized unidirectional effect of one variable on another. Arter. Scler = arteriolosclerosis; CVDA = atherosclerosis; Gross = gross infarcts; Hippo. Sclerosis = hippocampal sclerosis; Mesio Tangles = mesiotemporal tangles; Micro = microinfarcts; Neocort Tangles $=$ neocortical neurofibrilary tangles; TDP $=$ TAR DNAbinding protein $43 ; \mathrm{T}-\mathrm{EC}=$ neurofibrillary tangles in the entorhinal cortex; $\mathrm{T}$-Hip = neurofibrillary tangles in the hippocampus; T$\mathrm{IP}=$ neurofibrillary tangles in the inferior parietal cortex; T-MF = neurofibrillary tangles in the midfrontal cortex; TMT = neurofibrillary tangles in the midtemporal cortex.

This alternate parameterization was added to our a priori hypothesized path model (summarized with this modification in Fig 1) and was used in all subsequent SEM models involving this pathology. Use of alternate scorings for the cerebral infarct indicators did not appreciably change CFA results. Results of the CFA are available in Table s1.

After building these measurement models, we considered a series of structural models. Model 0 characterized the overall relation of age and dementia. Models 1 to 4 characterize four a priori hypothesized pathological pathways thought to mediate the association of age and dementia: a vascular pathway (model 1), an amyloid/tau pathway (model 2), a Lewy body disease pathway (model 3), and a TDP-43/hippocampal sclerosis pathway (model 4). Finally, we considered a full model (model 5; Fig 1) incorporating all four pathways and their hypothesized interrelations.

We conducted several sensitivity analyses. First, to consider other paths considered but excluded from our a priori model, we evaluated whether there was statistical justification for adding a path from infarcts to neuritic plaques, a path from neuritic plaques to hippocampal sclerosis, or a path from CAA to infarcts. Second, we used twogroup modeling to determine whether results varied across data source (MAP and ROS; MARS participants were excluded because of small numbers of MARS participants), across sex, or across persons from different birth cohorts. Two-group analyses of birth cohort addressed concerns that the distribution of pathologies could differ across those who died early or late relative to their peers. If so, then our sample would be enriched in pathological patterns characteristic of those who died prematurely and our results would not be generalizable. Thus, we defined a group born before 1915, who would be extremely unlikely to remain alive through the end of our sampling period and compared whether our structural model was consistent in those born before 1915 (who are representative of all dead individuals) and those born after 1915 (enriched in those who died prematurely). For each of our twogroup comparisons, we confirmed measurement invariance before proceeding. All statistical analyses were completed using Mplus (Version 8) or STATA (Version 14; StataCorp LP, College Station, TX). Throughout, we consider a $p$ value $<0.05$ to be statistically significant.

\section{Results}

The demographic and clinical characteristics of our sample are summarized in Table 1. On average, our sample was 89 years old at death (range, $65-108$ ). Of the $44 \%$ with a 
TABLE 1. Characteristics of the Eligible Sample Overall and by Dementia Status at Time of Death

\begin{tabular}{|c|c|c|c|}
\hline & Total & $\begin{array}{l}\text { Clinical Dementia } \\
\text { Diagnosis Preceding } \\
\text { Death }\end{array}$ & $\begin{array}{l}\text { No Clinical } \\
\text { Dementia Preceding } \\
\text { Death }\end{array}$ \\
\hline N (\%) & $1,362(100)$ & $594(44)$ & $768(56)$ \\
\hline Age at death, $y$, mean $(S D)$ & $88.6(6.7)$ & $90.0(6.2)$ & $87.5(6.9)$ \\
\hline Born before 1915, n (\%) & $389(29)$ & $212(36)$ & $177(23)$ \\
\hline Male, n (\%) & $462(34)$ & $187(31)$ & $275(36)$ \\
\hline Black, n (\%) & $53(4)$ & $23(4)$ & $30(4)$ \\
\hline \multicolumn{4}{|l|}{ Study, n (\%) } \\
\hline MAP & $670(49)$ & $268(45)$ & $401(52)$ \\
\hline ROS & $675(50)$ & $322(54)$ & $353(46)$ \\
\hline MARS & $17(1)$ & $4(1)$ & $13(2)$ \\
\hline Neuritic plaques, mean (SD) & $0.8(0.8)$ & $1.2(0.9)$ & $0.6(0.7)$ \\
\hline \multicolumn{4}{|c|}{ Neurofibrillary tangles, mean (SD) } \\
\hline Entorhinal cortex & $24.1(21.4)$ & $29.6(22.7)$ & $19.9(19.4)$ \\
\hline Hippocampus & $24.2(28.0)$ & $32.9(31.2)$ & $17.4(23.1)$ \\
\hline Midtemporal cortex & $6.0(12.0)$ & $10.7(15.5)$ & $2.4(6.4)$ \\
\hline Inferior parietal cortex & $2.6(6.8)$ & $4.8(9.0)$ & $0.9(3.8)$ \\
\hline Midfrontal cortex & $2.1(6.2)$ & $4.1(8.5)$ & $0.7(2.8)$ \\
\hline \multicolumn{4}{|l|}{ Gross infarcts, $\mathrm{n}(\%)$} \\
\hline 0 & $884(65)$ & $333(56)$ & $551(71)$ \\
\hline 1 & $243(18)$ & $116(20)$ & $127(17)$ \\
\hline$\geq 2$ & $233(17)$ & $143(24)$ & $90(12)$ \\
\hline \multicolumn{4}{|l|}{ Microinfarcts, n (\%) } \\
\hline 0 & $961(71)$ & $392(66)$ & $569(74)$ \\
\hline 1 & $244(18)$ & $117(20)$ & $127(17)$ \\
\hline$\geq 2$ & $155(11)$ & $83(14)$ & $72(9)$ \\
\hline \multicolumn{4}{|l|}{ Atherosclerosis, n (\%) } \\
\hline 0 (none) & $250(18)$ & $82(14)$ & $168(22)$ \\
\hline 1 & $632(47)$ & $271(46)$ & $361(47)$ \\
\hline 2 & $378(28)$ & $181(31)$ & $197(26)$ \\
\hline 3 (severe) & $93(7)$ & $56(9)$ & $37(5)$ \\
\hline \multicolumn{4}{|l|}{ Arteriolosclerosis, n (\%) } \\
\hline 0 (none) & $430(32)$ & $154(26)$ & $276(36)$ \\
\hline 1 & $462(34)$ & $201(34)$ & $261(34)$ \\
\hline 2 & $343(25)$ & $163(28)$ & $180(24)$ \\
\hline 3 (severe) & $114(8)$ & $71(12)$ & $43(6)$ \\
\hline
\end{tabular}


TABLE 1. Continued

\begin{tabular}{|c|c|c|c|}
\hline & Total & $\begin{array}{l}\text { Clinical Dementia } \\
\text { Diagnosis Preceding } \\
\text { Death }\end{array}$ & $\begin{array}{l}\text { No Clinical } \\
\text { Dementia Preceding } \\
\text { Death }\end{array}$ \\
\hline CAA, mean (SD) & $1.1(1.1)$ & $1.4(1.1)$ & $0.9(1.0)$ \\
\hline Neocortical Lewy bodies, n (\%) & $167(12)$ & $114(19)$ & $53(7)$ \\
\hline Hippocampal sclerosis, n (\%) & $119(9)$ & $94(16)$ & $25(3)$ \\
\hline TDP-43, mean (SD) & $0.6(1.0)$ & $0.4(0.7)$ & $1.0(1.2)$ \\
\hline
\end{tabular}

clinical dementia diagnosis preceding death, 85\% were diagnosed with $\mathrm{AD}$ dementia without a second cause of cognitive impairment, $11 \%$ were diagnosed with $\mathrm{AD}$ dementia with a second cause of cognitive impairment (eg, stroke), and 4\% were diagnosed with a non-AD primary cause of dementia. Brain pathology at autopsy was common regardless of dementia status. Age was significantly related to risk of dementia (odds ratio, 1.79; 95\% confidence interval, $1.51,2.12$ per 10 -year increase in age). This is represented by a standardized coefficient of 0.24 standard deviation $(\mathrm{SD})$ units (standard error $[\mathrm{SE}]=$ 0.03 ); see Table 2 (model 0 ).

\section{Model 1: Vascular Pathway}

To model the vascular pathway, we allowed the relation between age and dementia to be mediated by latent variables for vessel disease and infarcts (Fig 2; Table 2, model 1). Age had a significant direct effect on vessel disease, but not on infarcts. Vessel disease had a strong association with infarcts. Both vessel disease and infarcts had significant, independent associations with dementia. However, the direct effect of age on dementia remained large and significant (standardized coefficient $\beta=0.16$; $\mathrm{SE}=0.04$ ).

\section{Model 2: Amyloid/Tau Pathway}

Next, we considered an amyloid/tau pathway, allowing the relation between age and dementia to be mediated by neuritic plaques, our neocortical and mesiotemporal tangles latent variables, and CAA (Table 2, model 2). A priori, age was specified to have direct effects on neuritic plaques, neocortical tangles, mesiotemporal tangles, and CAA, in addition to its direct effect on dementia. Neuritic plaques were hypothesized to have direct effects on neocortical tangles, mesiotemporal tangles, and CAA. In turn, the two tangles latent variables and CAA were hypothesized to have direct effects on dementia. In considering potential modifications, the addition of a direct effect of neuritic plaques on dementia (Table 2, model 2a) improved model fit; we accepted the model with this modification as the final amyloid/tau model. Parameter estimates for model $2 \mathrm{a}$ are shown in Figure 3. Age had significant direct effects on neuritic plaques, mesiotemporal tangles, and CAA, but not neocortical tangles. Neuritic plaques had significant direct effects on neocortial tangles, mesiotemporal tangles, CAA, and dementia. The neocortial tangles latent variable had a significant effect on dementia, as did CAA, but the effect of the mesiotemporal tangles factor on dementia was nonsignificant. As with the vascular model, when considered independently, the amyloid model only partially mediated the effect of age on dementia, because the direct effect of age on dementia remained substantial and significant (standardized coefficient $\beta=0.21$; $\mathrm{SE}=0.03$ ).

\section{Model 3: Lewy Body Pathway}

Third, we considered a neocortical Lewy body pathway. We hypothesized that age would have direct effects on neuritic plaques, presence of neocortical Lewy body disease, and dementia; that neuritic plaques would have a direct effect on neocortical Lewy body disease; and the neocortical Lewy body measure would have a direct effect on dementia. This model had very poor fit (Table 2, model 3). The only possible modification was to include a direct effect of neuritic plaques on dementia. The modified model (Table 2, model 3a) was saturated and was accepted as the final Lewy body model. However, this result suggested that considering the Lewy body pathway separately from the amyloid/tau pathway or other hypothesized pathways involving neuritic plaques is inappropriate. Parameter estimates for model $3 \mathrm{a}$ are shown in Figure 4. Age had a small direct effect on neuritic plaques and a nonsignificant, essentially zero direct effect on Lewy body pathology. Neuritic plaques had significant effects on both Lewy body pathology and dementia. Lewy body disease had a substantial direct effect on dementia. As in 


\begin{tabular}{|c|c|c|c|c|c|c|c|c|}
\hline \multirow[b]{2}{*}{ Model } & \multirow[b]{2}{*}{ Description } & \multicolumn{3}{|c|}{ Statistical Index of Fit } & \multicolumn{4}{|c|}{ Practical Indices of Fit } \\
\hline & & $\chi^{2}$ & df & $p$ & RMSEA [CI] & CFI & TLI & $B_{\text {age }}(\mathrm{SE})$ \\
\hline 0 & Age, y & 0.00 & 0 & - & $0.000[0.000,0.000]$ & 1.000 & 1.000 & $0.24(0.03)$ \\
\hline 1 & Vascular & 2.97 & 5 & 0.71 & $0.000[0.000,0.028]$ & 1.000 & 1.011 & $0.16(0.04)$ \\
\hline 2 & Amyloid/tau hypothesized & 105.83 & 16 & $<0.0001$ & $0.064[0.053,0.076]$ & 0.986 & 0.968 & $0.19(0.03)$ \\
\hline $2 \mathrm{a}$ & Amyloid/tau, modified & 88.85 & 15 & $<0.0001$ & $0.060[0.048,0.072]$ & 0.988 & 0.972 & $0.21(0.03)$ \\
\hline 3 & Lewy body hypothesized & 101.53 & 1 & $<0.0001$ & $0.272[0.228,0.318]$ & 0.652 & 0.000 & $0.23(0.04)$ \\
\hline $3 a$ & Lewy body, modified & 0.00 & 0 & - & $0.000[0.000,0.000]$ & 1.000 & 1.000 & $0.21(0.03)$ \\
\hline 4 & TDP/HS hypothesized & 142.22 & 2 & $<0.0001$ & $0.227[0.196,0.2259]$ & 0.787 & 0.000 & $0.10(0.04)$ \\
\hline $4 a$ & TDP/HS modified & 2.16 & 1 & 0.14 & $0.029[0.000,0.084]$ & 0.998 & 0.982 & $0.11(0.04)$ \\
\hline 5 & All 4 pathways & 139.35 & 79 & $<0.001$ & $0.024[0.017,0.030]$ & 0.993 & 0.989 & $0.01(0.04)$ \\
\hline $5 a$ & $\begin{array}{l}\text { All } 4 \text { pathways nonsignificant } \\
\text { paths constrained to zero }\end{array}$ & 132.17 & 84 & $<0.001$ & $0.021[0.013,0.027]$ & 0.994 & 0.992 & $0.01(0.04)$ \\
\hline
\end{tabular}

Note: $\mathrm{N}=1,362$. For model names, $\mathrm{HS}=$ hippocampal sclerosis, and adjusted indicates that a direct effect of neuritic plaques on dementia was added to the a priori model. $\mathrm{B}_{\text {age }}(S E)$ = standardized regression coefficient (with standard error in parentheses) when predicting dementia from age. RMSEA = root mean square error of approximation; $\mathrm{CI}=$ confidence interval; CFI = comparative fit index; $\mathrm{TLI}=$ Tucker-Lewis index.

previous models, the direct effect of age on dementia (standardized coefficient $\beta=0.21 ; \mathrm{SE}=0.03$ ) remained large and significant, indicating that the neocortical Lewy body disease pathway only partially mediated the agedementia relation.

\section{Model 4: TDP-43/Hippocampal Sclerosis Pathway}

Fourth, we considered a pathway involving TDP-43 and hippocampal sclerosis. Because neuritic plaques were again implicated in TDP-43 and hippocampal sclerosis, these three variables were included a priori along with age and dementia. The a priori form of model 4 assumed that neuritic plaques would affect dementia only indirectly through its effects on TDP-43 and hippocampal sclerosis, but had poor fit to the data (Table 2, model 4). This again necessitated the addition of a direct path from neuritic plaques to dementia, resulting in model 4 a (Table 2, model 4a), which had very good fit and was accepted as the final model. However, as above, this indicated that considering TDP-43 and hippocampal sclerosis independent of other pathways involving neuritic plaques may not be

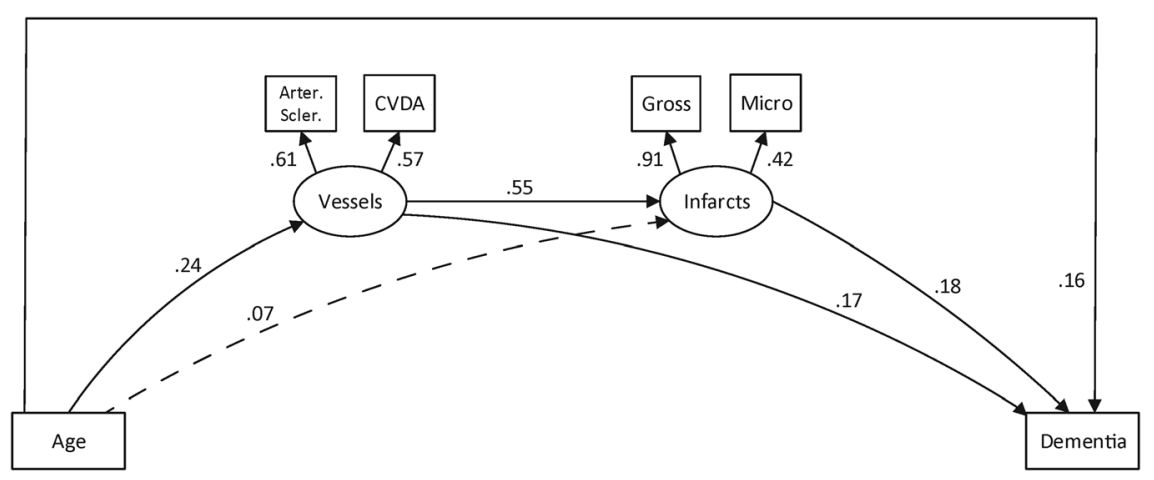

FIGURE 2: Results of path analysis considering mediation by the vascular pathology pathway of the effect of age on dementia risk. Squares or rectangles represent manifest variables and ellipses represent latent variables. Each single-headed arrow denotes a hypothesized unidirectional effect of one variable on another. Numbers associated with effects are standardized regression coefficients (eg, from age to dementia) or standardized factor loadings (ie, from a latent variable to its indicators). Paths that were statistically significant at $p<0.05$ are represented by solid lines. Paths that were hypothesized but were not statistically significant at $p<0.05$ are denoted by dashed lines. Arter. Scler $=$ arteriolosclerosis; CVDA $=$ atherosclerosis (cerebrovascular disease of the artery); Gross = gross infarcts; Micro = microinfarcts. 


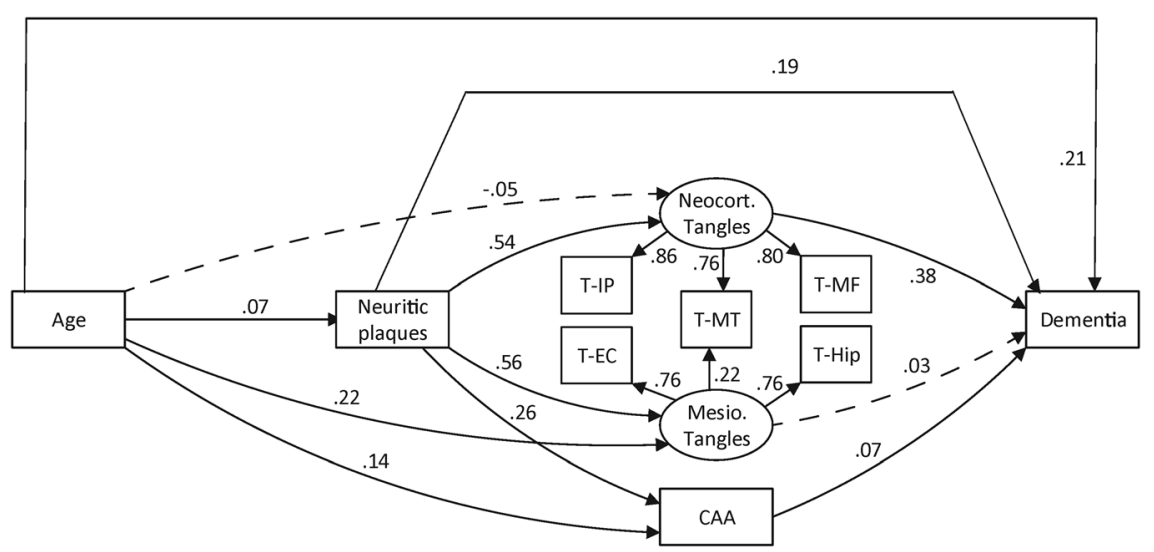

FIGURE 3: Results of path analysis considering mediation by the amyloid/tau pathway of the effect of age on dementia risk. Squares or rectangles represent manifest variables and ellipses represent latent variables. Each single-headed arrow denotes a hypothesized unidirectional effect of one variable on another. Numbers associated with effects are standardized regression coefficients (eg, from age to dementia) or standardized factor loadings (ie, from a latent variable to its indicators). Paths that were statistically significant at $p<0.05$ are represented by solid lines. Paths that were hypothesized but were not statistically significant at $p<0.05$ are denoted by dashed lines. Neocort Tangles $=$ neocortical neurofibrilary tangles; T-EC $=$ neurofibrillary tangles in the entorhinal cortex; T-Hip = neurofibrillary tangles in the hippocampus; T-IP = neurofibrillary tangles in the inferior parietal cortex; T-MF = neurofibrillary tangles in the midfrontal cortex; T-MT = neurofibrillary tangles in the midtemporal cortex.

appropriate. Parameter estimates for model 4a are shown in Figure 5. Age had direct effects on both TDP-43 and hippocampal sclerosis. Neuritic plaques had a significant effect on TDP-43. Both neuritic plaques and hippocampal sclerosis had relatively large effects on dementia, but the direct effect of TDP-43 on dementia was nonsignificant. However, the direct effect of age on dementia (standardized coefficient $\beta=0.11$; $\mathrm{SE}=0.04$ ) remained significant, indicating that the TDP-43/hippocampal sclerosis pathway did not fully mediate the age-dementia relation.

\section{Model 5: All Pathologies Combined}

We then considered a model incorporating all four pathways simultaneously (Fig 1). This model fit the data well (Table 2, model 5). However, five path coefficients were nonsignificant: the effects of age on infarcts, neocortical tangles, and Lewy body disease; the effect of vessels on neuritic plaques; and the effect of TDP-43 on dementia. When these five path coefficients were fixed at zero, the resulting model had slightly improved fit to the data (Table 2, model 5a). However, because this has little appreciable impact on the fit (Table 2), parameter estimates (Table s2), or percentage of the effect of age on dementia mediated by each path, we discuss model 5 here and in Figure 6.

Direct effects of variables on one another are similar to those discussed for the modeling of separate pathways, although the addition of a direct path between neuritic plaques and dementia, added to improve model fit in models $2 \mathrm{a}$, 3a, and 4a, was not necessary here. Model fit

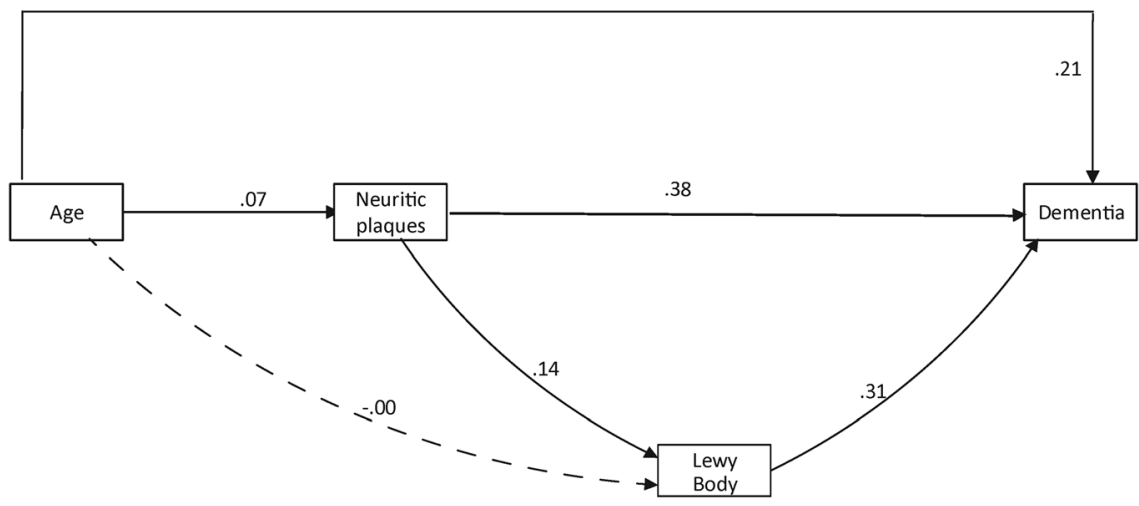

FIGURE 4: Results of path analysis considering mediation by the neocortical Lewy body pathology pathway of the effect of age on dementia risk. Squares or rectangles represent manifest variables and ellipses represent latent variables. Each single-headed arrow denotes a hypothesized unidirectional effect of one variable on another. Numbers associated with effects are standardized regression coefficients (eg, from age to dementia) or standardized factor loadings (ie, from a latent variable to its indicators). Paths that were statistically significant at $p<0.05$ are represented by solid lines. Paths that were hypothesized, but were not statistically significant at $p<0.05$ are denoted by dashed lines. 


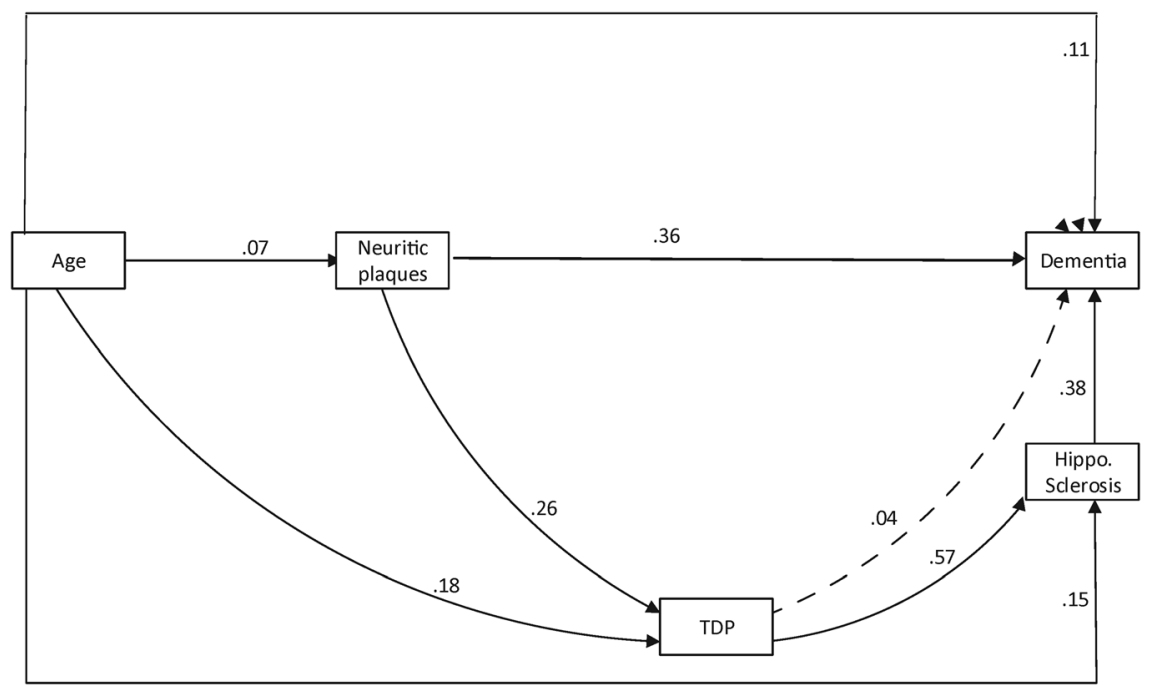

FIGURE 5: Results of path analysis considering mediation by the TDP43/hippocampal sclerosis pathway of the effect of age on dementia risk. Squares or rectangles represent manifest variables and ellipses represent latent variables. Each single-headed arrow denotes a hypothesized unidirectional effect of one variable on another. Numbers associated with effects are standardized regression coefficients (eg, from age to dementia) or standardized factor loadings (ie, from a latent variable to its indicators). Paths that were statistically significant at $p<0.05$ are represented by solid lines. Paths that were hypothesized but were not statistically significant at $p<0.05$ are denoted by dashed lines. Hippo. Sclerosis $=$ hippocampal sclerosis; TDP $=$ TAR DNA-binding protein 43.

worsened significantly if the path coefficients associated with any particular submodel (eg, the path coefficients contained in the vascular pathway) were fixed to zero. Similar worsening of model fit occurred if previously important individual path coefficients were fixed at zero. Unlike in the models for the individual pathways, the direct effect of age on dementia in model 5 was essentially zero (standardized coefficient, $\beta=0.01 ; \mathrm{SE}=0.04$ ) and nonsignificant; thus, the four pathways to dementia, taken together, fully mediated the relation between age and dementia. In this combined model, the vascular pathway accounted for $32 \%$ of the association between age and dementia, whereas the remaining three pathways, all of which involved neuritic plaques, accounted for $68 \%$ (the amyloid/tau pathway accounted for $24 \%$, the Lewy body pathway accounted for 1\%, and the TDP-43/hippocampal sclerosis pathway accounted for 43\%). (Recognizing that CAA can also be considered a vascular pathology, if we add the paths through CAA to our previously defined vascular pathway, these collectively account for $36 \%$ of the association between age and dementia.)

Sensitivity analyses were consistent with primary analyses. There was no evidence to support the addition of paths from infarcts to neuritic plaques, neuritic plaques to hippocampal sclerosis paths, or CAA to infarcts (all $p>0.3$ ). No significant differences in model 5 were found by sample source (MAP versus ROS) or by sex. ${ }^{37,38}$ When dividing our sample into those born before or after 1915 and allowing the path coefficients to be estimated freely, the statistical index of fit worsened notably $\left(\chi_{(176)}^{2}=276.97 ; p<0.001\right)$, but the model had excellent global fit (RMSEA, 0.029; CFI, 0.987; TLI, 0.983). Further analyses suggested allowing the path coefficient from neuritic plaques to neocortical tangles to differ across groups; the resulting model had improved statistical model fit $\left(\chi_{(24)}^{2}=23.62 ; p=0.48\right)$ and the standardized coefficient was $\beta=0.41$ ( $\mathrm{SE}=0.01$ ) for those born before 1915 and $\beta=0.57(\mathrm{SE}=0.01)$ for those born after 1915 , indicating a difference in magnitude, but not presence or direction of association, across groups.

\section{Discussion}

Overall, our results demonstrate that accumulation of known pathologies, including cerebrovascular disease, amyloid/tau, CAA, neocortical Lewy bodies, TDP-43, and hippocampal sclerosis, completely account for the increased risk of dementia with age. In the combined model, all four pathways remain important, indicating that each pathway uniquely contributes to the age-related increase in risk of dementia. Our models also suggest inter-relations between the amyloid/tau, Lewy body, and TDP-43/hippocampal sclerosis pathways. Specifically, neuritic plaques appear related to neurofibrillary tangles, CAA, neocortical Lewy body disease, and TDP-43, and after accounting for the association between neuritic plaques and these other pathologies, neuritic plaques had no direct effect on dementia. This suggests a significant contribution of amyloid beta to dementia through multiple pathways, not simply through influence on tangles. Importantly, although neuritic plaques appeared related to 


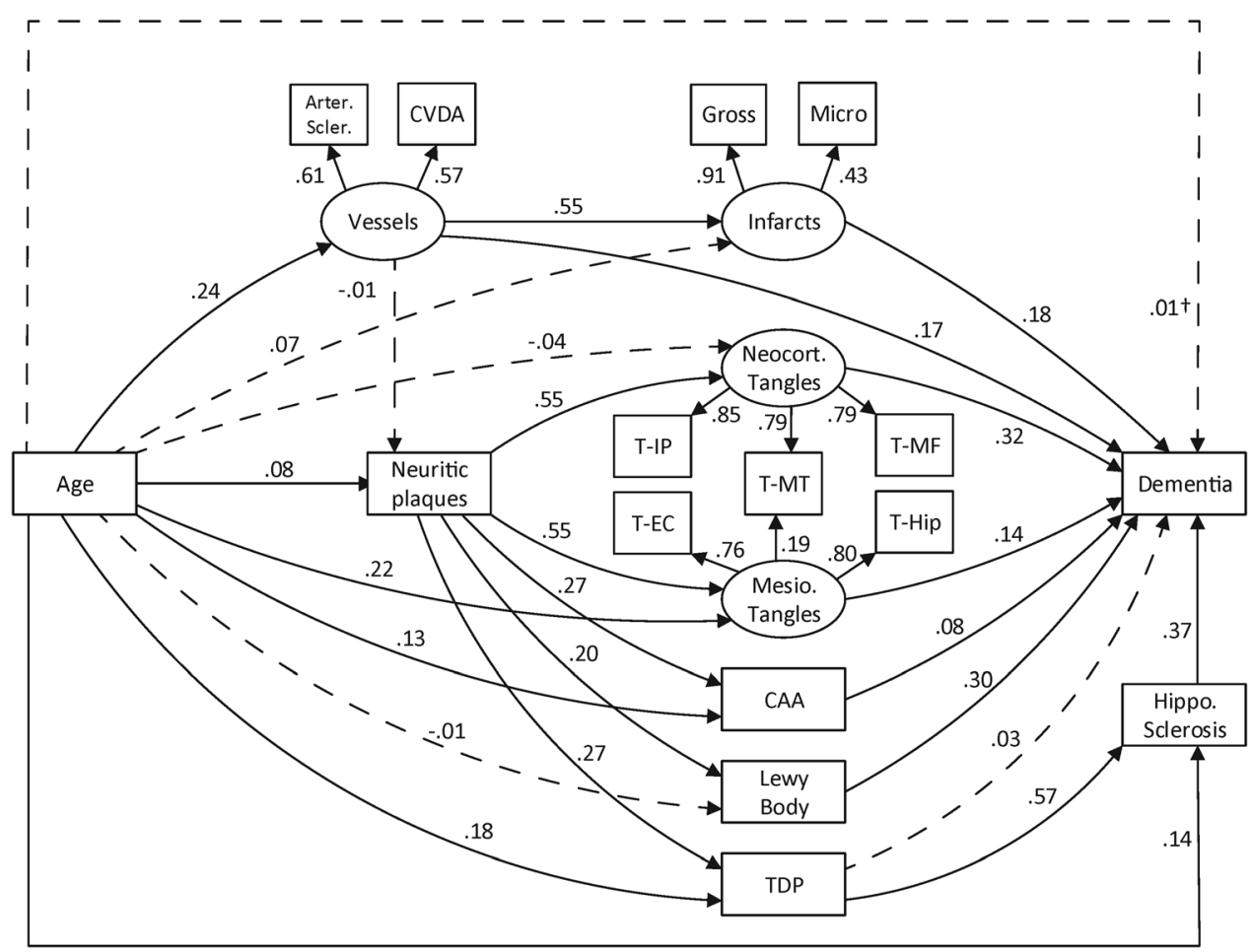

FIGURE 6: Results of path analysis of combined pathologic pathways mediating the effect of age on dementia risk. Squares or rectangles represent manifest variables and ellipses represent latent variables. Each single-headed arrow denotes a hypothesized unidirectional effect of one variable on another. Numbers associated with effects are standardized regression coefficients (e.g., from age to dementia) or standardized factor loadings (ie, from a latent variable to its indicators). Paths that were statistically significant at $p<0.05$ are represented by solid lines. Paths that were hypothesized but were not statistically significant at $p<0.05$ are denoted by dashed lines. Arter. Scler $=$ arteriolosclerosis; CAA = cerebral amyloid angiopathy; CVDA $=$ atherosclerosis; Gross $=$ gross infarcts; Hippo. Sclerosis $=$ hippocampal sclerosis; Micro = microinfarcts; Mesio. Tangles = mesiotemporal neurofibrilary tangles; Neocort Tangles = neocortical neurofibrilary tangles; TDP = TAR DNA-binding protein 43; T-EC = neurofibrillary tangles in the entorhinal cortex; T-Hip = neurofibrillary tangles in the hippocampus; T$\mathrm{IP}=$ neurofibrillary tangles in the inferior parietal cortex; $\mathrm{T}-\mathrm{MF}=$ neurofibrillary tangles in the midfrontal cortex; $\mathrm{T}$ MT = neurofibrillary tangles in the midtemporal cortex.

CAA and TDP-43/hippocampal sclerosis, these pathologies also appear to mediate the elevated risk of dementia on age through pathways that do not involve neuritic plaques. Finally, our model supports independence between the collective influence of the amyloid/tau, Lewy body, and TDP-43/hippocampal sclerosis pathways from our vascular pathway, with the vascular pathway accounting for $32 \%$ of the variance in dementia risk attributable to age and the remaining pathways collectively accounting for $68 \%$.

This work is generally consistent with previous work. Multiple studies have shown that vascular, amyloid/ tau, Lewy bodies, and TDP-43/hippocampal sclerosis pathologies all contribute to cognition in older ages. ${ }^{8}$ Interestingly, despite suggestion of a synergistic relation in other contexts, ${ }^{12}$ our work confirms previous work in ROS/MAP and elsewhere suggesting that vascular pathology does not interact with $\mathrm{AD}$ pathology to promote dementia. ${ }^{3,39-41}$ Nonetheless, the relative contribution of various pathological pathways may differ by cognitive outcome. For example, in previous work in ROS/MAP, a vascular pathway accounted for $20 \%$ of variance in the association between age at death and predeath decline in episodic memory and $29 \%$ of the variance in the corresponding association with decline in nonepisodic memory. ${ }^{42}$

Little is known about the impact of TDP-43 and hippocampal sclerosis on cognition and dementia, alone or in the context of other pathologies. In the current analysis, the effect of TDP-43 on dementia risk was completely mediated by hippocampal sclerosis. However, we and others have reported that TDP-43 is independently associated with worse episodic memory, cognitive decline, and increased odds of dementia. ${ }^{7,43,44}$ The source of this discrepancy remains unknown. Potential reasons include the omission of other pathologies in previous work, unique or synergistic effects of hippocampal sclerosis and TDP-43 on domain-specific cognition, or the sparsity of persons in our sample with hippocampal sclerosis but no TDP-43 $(\mathrm{n}=11)$. Because hippocampal sclerosis has been reported to be segmental, ${ }^{45}$ neuropathological assessment of multiple sections of hippocampus may be necessary to fully 
characterize the condition. Regardless, further work on the relation among $\mathrm{AD}, \mathrm{TDP}-43$, and hippocampal sclerosis is warranted, particularly given that pathways involving these pathologies appear to mediate a large percentage of the age-related increase in dementia risk.

Our study extends previous attempts to characterize the collective impact of multiple pathologies on cognition in several important ways. Our study included all major pathologies thought to contribute to dementia risk, which, combined with an SEM approach, allowed us to describe and quantify the relative contributions of hypothesized pathways, not just individual predictors. Notably, use of SEM allowed us to think globally about complex causal relationships between pathologies, enabled by the use of latent variables, simultaneous consideration of individual variables as both predictors of an outcome and mediators of a different predictor-outcome relation, and established methods to assess overall motel fit. Our approach also addresses the novel question of whether the collective influence of known pathologies can account for the association between age and dementia risk. Previous studies have frequently considered only a subset of pathologies, taken a different approach, or addressed related, but distinct, questions. For example, in previous work in ROS/MAP, global Alzheimer pathology, amyloid, tangles, gross infarcts, microinfarcts, and neocortical Lewy bodies accounted for $41 \%$ of the total variance in the rate of $\operatorname{cog}$ nitive decline preceding to death. ${ }^{32}$ Although this study was limited by omission of TDP-43, hippocampal sclerosis, and measures of vessel disease, it appears unlikely that $100 \%$ of the variance would be accounted for after inclusion of these additional factors. However, the conclusion that pathology cannot fully account for variation in cognitive decline is not necessarily at odds with our findings here. Whereas we conclude that accumulating pathology fully mediated the elevation in risk of dementia observed with age, we did not address the related question addressed in this previous study of whether differences in pathology account for variance in measures of cognition among similarly aged persons.

This study had many strengths. Our large clinicopathological sample was recruited from the community before the known onset of dementia, and autopsies were performed on the vast majority of deceased participants. In addition, our conclusions were consistent across data sources, birth cohorts, and sex. Our results are arguably generalizable to the white general population. The study also benefited from high-quality clinicopathological data quantifying all the major dementia-related pathologies and study-based clinical dementia ascertainment derived from in-person examinations, which is less prone to misclassification than ascertainment based on medical records or similar sources.
This study also has limitations. Some degree of misclassification is expected. However, despite expected misclassification, we were able to account for all the variance in clinical dementia status associated with increasing age. Our data were cross-sectional. Thus, we cannot prove the temporal ordering or causal relations implied by our model. We can only conclude that our hypothesized model is or is not consistent with the data; we cannot conclude that we have identified the true underlying causal relations. Other hypothesized models may also fit the data and may or may not better reflect the true underlying causal structure connecting age, neuropathology, and dementia. Our results may not be generalizable to all race/ethnic groups. Whether there are differences in prevalence of brain pathologies across race/ethnic groups remains unclear ${ }^{46-48}$ and whether such differences would translate into differences in the relative importance of particular pathways remains unknown. Similar work in more diverse samples is warranted. The relative contribution of various pathologic pathways may differ by cognitive outcome $^{42}$ or by presence of specific genetic polymorphisms. ${ }^{49,50}$ Future work should evaluate this possibility. Finally, we considered broad characterizations of neuropathological pathways. More detailed models (eg, a previous work ${ }^{51}$ ) may reveal further insights. In particular, our models do not account for all vascular pathologies (eg, microbleeds, damaged neurovascular unit, etc.) or functional/molecular processes (eg, contractility, glymphatic drainage, etc.).

Our findings have significant implications for clinical trial design and drug discovery. Efforts to develop therapies for $\mathrm{AD}$ have long targeted amyloid. Whereas our analyses support the supposition that an effective antiamyloid medication may have benefit on multiple pathological pathways (if amyloid is truly causal and if treatment is given when maximally effective, likely decades preceding clinical symptoms), our results also suggest that it would be far from a panacea. Other pathologies clearly have independent and substantial contributions to dementia risk, even in our sample where the vast majority of cases were clinically diagnosed with $\mathrm{AD}$ and most had pathological evidence of AD. These other pathologies should be considered valid therapeutic targets. Given the hypothesized complex pathways underlying dementia, a "one-size-fits-all" therapeutic strategy is unlikely to be successful. Drug discovery work, including clinical trials, will face numerous challenges in identifying persons likely to benefit from a given therapy, adequately powering studies to demonstrate benefit among this subgroup, and managing toxicity and cost considerations for patients with evidence of multiple pathological processes. 


\section{Acknowledgment}

This work was conceived and begun at the Friday Harbor Conference on Advanced Psychometric Methods in Aging Research; this conference is funded by $\mathrm{NIH}$ (R13AG030995). This work was supported by grants from NIH (R13AG030995, P30AG010161, R01A017917, RF1AG022018, R01AG042210, R01AG034374, and RFAG015819). Dr Bangen was funded by VA Clinical Science Research \& Development (Career Development Award-2 1IK2CX000938) and the Alzheimer's Association (NIRG-15-364251). Dr James was supported by NIH K01AG050823. Dr Delano-Wood was funded by the Alzheimer's Association (NIRG-15-364251). Dr Lamar was funded by K01-AG040192 from the National Institute on Aging. Dr Gross was funded by K01-AG050699 from the National Institute on Aging. Dr Lim was funded by the NHMRC-ARC Dementia Research Development Fellowship (GNT1111603). Dr Zahodne was funded by NIA (R00 AG047953, R01 AG054520).

We thank the participants, staff, and funders that make this work possible.

\section{Author Contributions}

M.P., E.M., A.S., B.J., L.Y., N.A., K.B., L.D., M.L., Y.L., K.N., L.Z., A.G., D.M., K.W., and J.S. contributed to the conception and design of the study. B.J., A.G., M.P., J.S., K.W., and L.Y. contributed to the acquisition and analysis of the data. B.J., E.M., M.P., A.S., J.S., and K.W. contributed to drafting the text or preparing figures.

\section{Potential Conflicts of Interest}

Nothing to report.

\section{References}

1. Suemoto CK, Ferretti-Rebustini RE, Rodriguez RD, et al. Neuropathological diagnoses and clinical correlates in older adults in Brazil: a cross-sectional study. PLoS Med 2017;14: e1002267.

2. Tanskanen M, Makela M, Notkola IL, et al. Population-based analysis of pathological correlates of dementia in the oldest old. Ann Clin Transl Neurol 2017;4:154-165.

3. White LR, Edland SD, Hemmy LS, et al. Neuropathologic comorbidity and cognitive impairment in the Nun and Honolulu-Asia Aging Studies. Neurology 2016;86:1000-1008.

4. Schneider JA, Arvanitakis Z, Bang W, Bennett DA. Mixed brain pathologies account for most dementia cases in community-dwelling older persons. Neurology 2007;69:2197-2204.

5. Rahimi J, Kovacs GG. Prevalence of mixed pathologies in the aging brain. Alzheimers Res Ther 2014;6:82.

6. Jellinger KA, Attems J. Challenges of multimorbidity of the aging brain: a critical update. J Neural Transm (Vienna) 2015;122:505-521.
7. James BD, Wilson RS, Boyle PA, et al. TDP-43 stage, mixed pathologies, and clinical Alzheimer's-type dementia. Brain 2016;139: 2983-2993.

8. Kapasi A, DeCarli C, Schneider JA. Impact of multiple pathologies on the threshold for clinically overt dementia. Acta Neuropathol 2017;134:171-186.

9. Kawas $\mathrm{CH}$, Kim RC, Sonnen JA, et al. Multiple pathologies are common and related to dementia in the oldest-old: The $90+$ Study. Neurology 2015;85:535-542.

10. Corrada MM, Berlau DJ, Kawas CH. A population-based clinicopathological study in the oldest-old: The $90+$ Study. Curr Alzheimer Res 2012;9:709-717.

11. Pathological correlates of late-onset dementia in a multicentre, community-based population in England and Wales. Neuropathology Group of the Medical Research Council Cognitive Function and Ageing Study (MRC CFAS). Lancet 2001;357:169-175.

12. Snowdon DA, Greiner LH, Mortimer JA, et al. Brain infarction and the clinical expression of Alzheimer disease. The Nun Study. JAMA $1997 ; 277: 813-817$.

13. Corrada MM, Sonnen JA, Kim RC, Kawas CH. Microinfarcts are common and strongly related to dementia in the oldest-old: The 90 + Study. Alzheimers Dement 2016;12:900-908.

14. Charidimou A, Gang Q, Werring DJ. Sporadic cerebral amyloid angiopathy revisited: recent insights into pathophysiology and clinical spectrum. J Neurol Neurosurg Psychiatry 2012;83:124-137.

15. Case NF, Charlton A, Zwiers A, et al. Cerebral amyloid angiopathy is associated with executive dysfunction and mild cognitive impairment. Stroke 2016;47:2010-2016.

16. Boyle PA, Yu L, Nag S, et al. Cerebral amyloid angiopathy and cognitive outcomes in community-based older persons. Neurology 2015;85:1930-1936.

17. Hamilton RL. Lewy bodies in Alzheimer's disease: a neuropathological review of 145 cases using alpha-synuclein immunohistochemistry. Brain Pathol 2000;10:378-384.

18. Schneider JA, Arvanitakis Z, Leurgans SE, Bennett DA. The neuropathology of probable Alzheimer disease and mild cognitive impairment. Ann Neurol 2009;66:200-208.

19. Walker Z, McKeith I, Rodda J, et al. Comparison of cognitive decline between dementia with Lewy bodies and Alzheimer's disease: a cohort study. BMJ Open 2012;2:e000380.

20. Chung EJ, Babulal GM, Monsell SE, et al. Clinical features of Alzheimer disease with and without Lewy bodies. JAMA Neurol 2015;72: 789-796.

21. Connor DJ, Salmon DP, Sandy TJ, et al. Cognitive profiles of autopsy-confirmed Lewy body variant vs pure Alzheimer disease. Arch Neurol 1998;55:994-1000.

22. Amador-Ortiz C, Lin WL, Ahmed Z, et al. TDP-43 immunoreactivity in hippocampal sclerosis and Alzheimer's disease. Ann Neurol 2007;61: 435-445.

23. Nelson PT, Schmitt FA, Lin Y, et al. Hippocampal sclerosis in advanced age: clinical and pathological features. Brain 2011;134(pt 5):1506-1518.

24. Josephs KA, Whitwell JL, Knopman DS, et al. Abnormal TDP-43 immunoreactivity in $A D$ modifies clinicopathologic and radiologic phenotype. Neurology 2008;70(19 pt 2):1850-1857.

25. Bennett DA, Schneider JA, Arvanitakis Z, Wilson RS. Overview and findings from the religious orders study. Curr Alzheimer Res 2012;9: 628-645.

26. Bennett DA, Schneider JA, Buchman AS, et al. Overview and findings from the Rush Memory and Aging Project. Curr Alzheimer Res 2012;9:646-663.

27. Barnes LL, Shah RC, Aggarwal NT, Bennett DA, Schneider JA. The Minority Aging Research Study: ongoing efforts to obtain brain 
donation in African Americans without dementia. Curr Alzheimer Res 2012;9:734-745

28. McKhann G, Drachman D, Folstein M, et al. Clinical diagnosis of Alzheimer's disease: report of the NINCDS-ADRDA Work Group under the auspices of Department of Health and Human Services Task Force on Alzheimer's Disease. Neurology 1984;34:939-944.

29. Roman GC, Tatemichi TK, Erkinjuntti T, et al. Vascular dementia: diagnostic criteria for research studies. Report of the NINDS-AIREN International Workshop. Neurology 1993;43:250-260.

30. Bennett DA, Schneider JA, Aggarwal NT, et al. Decision rules guiding the clinical diagnosis of Alzheimer's disease in two community-based cohort studies compared to standard practice in a clinic-based cohort study. Neuroepidemiology 2006;27:169-176.

31. McKeith IG, Perry EK, Perry RH. Report of the second dementia with Lewy body international workshop: diagnosis and treatment. Consortium on Dementia with Lewy Bodies. Neurology 1999;53:902-905.

32. Boyle PA, Wilson RS, Yu L, et al. Much of late life cognitive decline is not due to common neurodegenerative pathologies. Ann Neurol 2013;74:478-489.

33. Boyle PA, Yang J, Yu L, et al. Varied effects of age-related neuropathologies on the trajectory of late life cognitive decline. Brain 2017; 140:804-812.

34. McKeith IG, Galasko D, Kosaka K, et al. Consensus guidelines for the clinical and pathologic diagnosis of dementia with Lewy bodies (DLB): report of the Consortium on DLB International Workshop. Neurology 1996;47:1113-1124.

35. Schneider JA, Arvanitakis Z, Yu L, et al. Cognitive impairment, decline and fluctuations in older community-dwelling subjects with Lewy bodies. Brain 2012;135(pt 10):3005-3014.

36. Browne M, Cudeck R. Alternative ways of assessing model fit. In: Bollen K, Long J, eds. Testing Structural Equation Models. Newbury Park, CA: Sage; 1993:136-162.

37. Widaman KF, Reise SP. Exploring the measurement invariance of psychological instruments: Applications in the substance use domain. The science of prevention: methodological advances from alcohol and substance abuse research. Washington, DC: American Psychological Association; 1997:281-324.

38. Muthén LK, Muthén BO. Mplus User's Guide, 8th ed. Los Angeles, CA: Muthén \& Muthén; 1998-2017.
39. Schneider JA, Boyle PA, Arvanitakis Z, Bienias JL, Bennett DA. Subcortical infarcts, Alzheimer's disease pathology, and memory function in older persons. Ann Neurol 2007;62:59-66.

40. Troncoso JC, Zonderman AB, Resnick SM, et al. Effect of infarcts on dementia in the Baltimore Longitudinal Study of Aging. Ann Neurol 2008;64:168-176.

41. Sonnen JA, Larson EB, Crane PK, et al. Pathological correlates of dementia in a longitudinal, population-based sample of aging. Ann Neurol 2007;62:406-413.

42. Yu L, Boyle PA, Leurgans S, Schneider JA, Bennett DA. Disentangling the effects of age and APOE on neuropathology and late life cognitive decline. Neurobiol Aging 2014;35:819-826.

43. Josephs KA, Whitwell JL, Weigand SD, et al. TDP-43 is a key player in the clinical features associated with Alzheimer's disease. Acta Neuropathol 2014;127:811-824.

44. Nag S, Yu L, Capuano AW, et al. Hippocampal sclerosis and TDP-43 pathology in aging and Alzheimer disease. Ann Neurol 2015;77: 942-952.

45. Ighodaro ET, Jicha GA, Schmitt FA, et al. Hippocampal sclerosis of aging can be segmental: two cases and review of the literature. J Neuropathol Exp Neurol 2015;74:642-652.

46. Graff-Radford NR, Besser LM, et al. Neuropathological differences by race from the National Alzheimer's Coordinating Center. Alzheimers Dement 2016;12:669-677.

47. Wilkins $\mathrm{CH}$, Grant EA, Schmitt SE, McKeel DW, Morris JC. The neuropathology of Alzheimer disease in African American and white individuals. Arch Neurol 2006;63:87-90.

48. Sandberg G, Stewart W, Smialek J, Troncoso JC. The prevalence of the neuropathological lesions of Alzheimer's disease is independent of race and gender. Neurobiol Aging 2001;22:169-175.

49. Bennett DA, Wilson RS, Schneider JA, et al. Apolipoprotein E epsilon4 allele, $A D$ pathology, and the clinical expression of Alzheimer's disease. Neurology 2003;60:246-252.

50. Kasanuki K, Heckman MG, Diehl NN, et al. Regional analysis and genetic association of nigrostriatal degeneration in Lewy body disease. Mov Disord 2017;32:1584-1593.

51. Mungas D, Tractenberg R, Schneider JA, Crane PK, Bennett DA. A 2-process model for neuropathology of Alzheimer's disease. Neurobiol Aging 2014;35:301-308. 